\title{
Simulation Study on Classroom Crowd Evacuation Under Panic Emotion Infection
}

\author{
Linna $\mathrm{LI}^{\mathrm{a}}$ and Baoyun WANG ${ }^{\mathrm{b}, 1}$

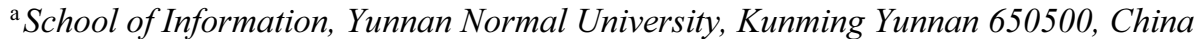 \\ ${ }^{\mathrm{b}}$ School of Mathematics, Yunnan Normal University, Kunming Yunnan 650500, China
}

\begin{abstract}
In case of emergency, the classroom is prone to crowd congestion accidents. In order to explore the impact of crowd panic emotion infection on evacuation and the role of the manager in the process of emotional infection, the model was simulated by using unity3D software, and the role of the model in different scenarios was analyzed to describe the evacuation phenomenon of the group under panic emotion. The results show that the presence of administrators can improve evacuation efficiency and reduce the rate of emotional infection. Extroverted individuals can accelerate the speed of emotional infection; The fewer individuals in the classroom, the slower the emotional infection, the higher the evacuation efficiency.
\end{abstract}

Keywords. Emotional contagion model, ECEM model;panic, classroom, Crowded.

\section{Introduction}

In recent years, the problem of crowd evacuation based on individual mood changes has received a lot of attention, and panic infection has become a hot spot in the field of crowd evacuation research. Infection of panic refers to that when an emergency occurs, under the condition of lack of sense of security, the panic of other individuals will affect their judgment ability due to the effect of suggestion and infection, leading to the expansion of their panic. Under the influence of other individuals, individuals will imitate others, and the deep panic of other individuals will also enlarge their panic[1] Panic will directly affect evacuation activities[2], which is embodied in individual speed and direction. At present, many scholars have integrated emotional infection model into the process of crowd emergency. Wang Ruixiang et al.[3]improved the spread model of infectious diseases and added intervention factors into the panic psychological spread model to study the inhibition effect of intervention measures on the spread of panic. Cao Mengxiao et al.[4] established Personalized Durupinar (P-Durupinar) model based on Durupinar model, which confirmed the importance of Personalized influence on emotion infection in crowd movement. Liu Cuijuan et al.[5]proposed a multi-agent emotional infection model (ECEM) based on OCEAN model, which combined agent movement with density and personality, and verified the authenticity of non-contact emotional infection. Liu Zhen et al. [6] proposed a calculation method of emotional infection based on whether

\footnotetext{
${ }^{1}$ Corresponding Author, Baoyun WANG, School of Mathematics, Yunnan Normal University, Kunming, Yunnan, China. E-mail:wspbmly@163.com.
} 
there are managers in the crowd, and demonstrated the restraining effect of managers on panic in the crowd. Liu Tingting et al.[7]proposed a simulation model of crowd trample based on psychological principles to simulate the process of crowd trample, and provided the judgment method of crowd trample, which was verified by the simulation of crowd trample on the footbridge.

According to statistics, schools account for $20.97 \%$ of all places where stampedes occur[8], so how to avoid stampedes on campus has become the focus of attention, among which classroom crowd evacuation is the most typical scene. Many scholars have conducted simulation studies on classroom model crowd evacuation $[9,10,11]$, most of which focus on the impact of classroom structure on evacuation, such as odd and even exits, seat arrangement, exit width and other factors, and seldom consider the impact of panic on classroom crowd evacuation. Based on the research of Liu Cuijuan and Liu Zhen, this paper conducts a simulation study on classroom crowd evacuation under panic.

\section{Panic Infection}

In this paper, the ECEM model[5]proposed by Liu Cuijuan and the emotional infection model with administrator[6]proposed by Liu Zhen were applied to the classroom scene to conduct simulation modeling of panic emotion infection in the crowd.

\subsection{Panic Contagion Model}

In the ECEM model, the influence of personality and distance on emotion was considered. On the one hand, the classification of individual personality, different types of individuals have different ability to receive emotional infection. Based on OCEAN personality theory, the model divides individuals into five types: open individuals $(\mathrm{O})$, conscientious individuals (C), extroverted individuals (E), agreeableness individuals (A), and neurotic individuals $(\mathrm{N})$. On the other hand, risk factors will stimulate individual panic, the closer to the source of danger, the higher the individual's emotional value.

The emotional infection rules in the model are divided into the following three points: Rule 1. Emotional infection is transmitted from individuals with high emotional value to individuals with low emotional value. Rule 2. Emotional contagion occurs within the detection area of the individual (i.e. the radius of emotional contagion). Rule 3. During the process of emotional infection, individual emotions gradually increase.

In order to explore the influence of administrators (teachers) in the process of panic infection, panic attenuation parameters were added into the ECEM model. The manager is equivalent to an inhibitor of panic emotions. When the emotional value of the individual differs greatly from that of the manager, the individual's emotional attenuation will be larger, and he will be inhibited more panic emotions. In the actual situation, teachers will pay more attention to students with high panic value, while students with low panic value do not need excessive attention from teachers.

\subsection{Parameters of Panic Emotion Infection Model}

- (1) Individual parameter definition 
In the panic emotion infection model, each individual defines four variables $\left\{\delta_{i}, \varepsilon_{j}, d_{\max }, R\right\}$, as shown in table 1 .

Table 1. Parameter definition of panic emotion infection model.

\begin{tabular}{cc}
\hline variate & definition \\
\hline$\delta_{i}$ & Emotional receptivity \\
$\varepsilon_{j}$ & Emotion-sending capacity \\
$d_{\max }$ & Radius of emotional contagion \\
$R$ & Radius of the individual \\
\hline
\end{tabular}

The emotional value is calculated as follows ${ }^{[5]}$ :

$$
\begin{aligned}
& P_{i}(t+1)=P_{i}(t)+\sum_{j=1}^{m} G(i, j)+D(i, j) \\
& G(i, j)=\delta_{i} * \varepsilon_{j} *\left(1-\frac{d_{i j}}{d_{\max }}\right) * P_{i}(t) \\
& D(i, d)=e^{\left(\frac{\left\|r_{i}-r_{d}\right\| \mid}{2 R}\right)}
\end{aligned}
$$

In the above formula, $P_{i}(t)$ is the emotional value of individual $\mathrm{i}$ at time $\mathrm{t}$; $G(i, j)$ and $D(i, j)$ are the influence of personality and distance on emotion respectively; $\mathrm{j}$ is the neighbor whose $P_{i}<P_{j}$, m is the number of neighbor individuals whose $P_{i}<P_{j} ; d_{i j}$ is the Euclidean distance between individual $\mathrm{i}$ and individual $\mathrm{j}$; $\left\|r_{i}-r_{d}\right\|$ is the distance between individual $\mathrm{i}$ from hazards.

- (2) Definition of attenuation parameters of panic

In the case of a supervisor, the scope of influence is the whole classroom, that is, when the teacher is present, all the students' emotions can be affected. The manager's emotional value, $P_{t e}$, remained constant throughout the evacuation.

Let $P_{i}$ be the emotional intensity of an individual. If $P_{t e}<P_{i}$, emotional value of individual panic emotion is[6]:

$$
\Delta P_{e}=\frac{P_{t e}-\left(1-P_{i}\right)}{P_{t e}+\left(1-P_{i}\right)}
$$

At this point, the individual's emotional value is:

$P_{i}-=\Delta P_{e}$

In the formula, $\Delta P_{e}$ is the emotional value of individual panic emotion; $P_{t e}$ is the emotional value of the manager; $P_{i}$ is the emotional value of individual $i$.

\subsection{Panic Emotion Infection Model Algorithm Flow}

Algorithm 1 algorithm of panic emotion infection model

Input: $\delta_{i}, \varepsilon_{i}, d_{\max }, R, P_{t e}$

Output: $P_{i}$ 
Step 1 Initialize individual mood values.

Step 2 For all individuals, if the individual emotional value $\mathrm{P}<1$, other individuals within the infection radius of the individual were detected.

Step 3 For neighbor $\mathrm{j}$, if $P_{i}<P_{j}$, using values of $\delta_{i}, \varepsilon_{i}, d_{\text {max }}, R, P_{t e}$, and according to the type (1), (2), (3), (4), (5) computing $P_{i}$.

Step 4 If the individual emotion value $\mathrm{P} \geq 1$, the emotion value $\mathrm{P}$ is updated to 1 .

Algorithm 2 Emotion and motion coupling algorithm

Input: $V_{n}$

Output: $V_{i}$

Step 1 Assign the initial speed $V_{n}$ for all individuals, $V_{n}=1.35 \mathrm{~m} / \mathrm{s}$

Step 2 Calculate the current position of the individual, and update the density if the agent does not reach the target point.

Step 3 According to algorithm 1 update the emotional value $P_{i}$ of individual i.

Step 4 Based on the current emotional value and density update individual speed.

Among them, density and speed updates[5] are as follows:

$$
\begin{aligned}
\rho & =\frac{N}{S} \\
V_{i} & =\left(1+P_{i}(t)\right) * \frac{V_{n}}{\rho_{i}}
\end{aligned}
$$

In the formula, $\rho$ is the density around the individual; $\mathrm{N}$ is the number of individuals within the detection range; $\mathrm{S}$ is the area of individual detection range; $V_{i}$ is the actual speed of the individual; $V_{n}$ is the initial velocity of the individual.

\section{Experiment and Analysis}

The software used in this experiment is Unity3D and Visual Studio 2019. The experimental results are divided into three parts: 1) The influence of having and having no manager on emotional infection and crowd movement; 2) The influence of different personality ratio on emotional infection; 3) The influence of different number of individuals on emotional infection and crowd movement in the absence of managers. In the experiment, the $\delta$ values of emotional infection receptivity of individuals with different personalities [5] are as follows: For O-type individuals, the value is 0.35 , For Ctype individuals, the value is 0.20 , For E-type individuals, the value is 0.15 , For A-type individuals, the value is 0.30 , and for N-type individuals, the value is 0.15 . 


\subsection{Scene Setting}

The simulation scene is a classroom $8 \mathrm{~m}$ long and $7 \mathrm{~m}$ wide. The size of the indoor desk is $0.6 \mathrm{~m}^{*} 0.4 \mathrm{~m}^{*} 0.5 \mathrm{~m}$, the outlet is $1 \mathrm{~m}$ wide. According to the actual earthquake monitoring, students fled from the front door during earthquake evacuation, so only one exit was set up in the scene. The classroom layout is shown in figure 1 . Where, the radius of the individual is $0.19 \mathrm{~m}[12]$, according to the highest density of 9 people $/ \mathrm{m}^{2}$ in crowd crowding, the radius of emotional infection is $0.56 \mathrm{~m}$.

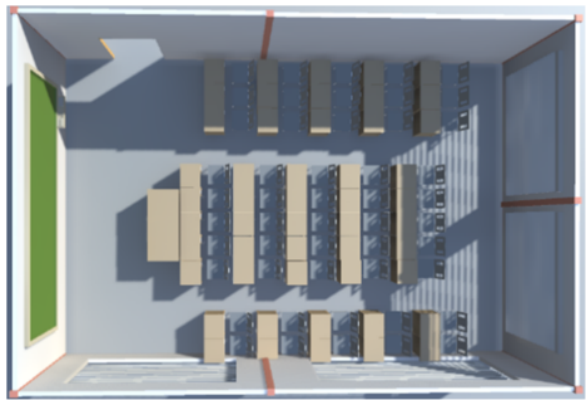

Figure 1. Classroom layout.

\subsection{Analysis of Experimental Results}

(1) Experiment 1: Administrator or no administrator

In order to verify the influence of teachers on the speed of emotional infection and evacuation, the number of experimental agents is set as follows: In the case of no teacher, 50 agents were set in the classroom scene. In the case of teachers, set up 51 agents. Figure 2 and figure 3 show the comparison of evacuation speed and emotional infection speed with and without teachers.

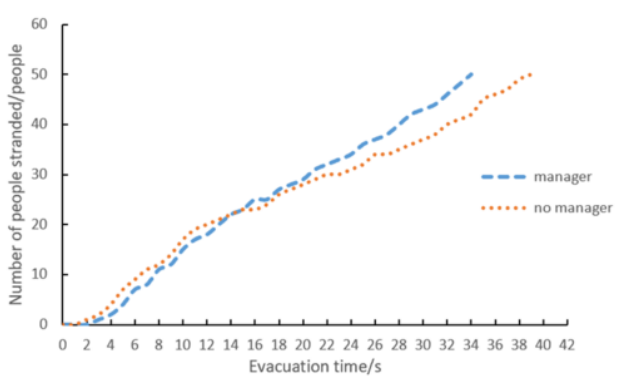

Figure 2. Evacuation time with or without a manager. 


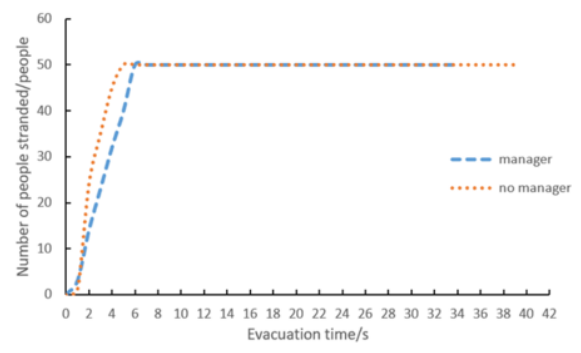

Figure 3. The rate of emotional infection with or without a manager.

Analyze the results of figure 2 and figure 3: Comparison of evacuation speed is shown in figure 2. At the initial stage of evacuation, the evacuation speed of students without teachers is faster than that with teachers. After $14 \mathrm{~s}$, the evacuation speed with the teacher is higher than that without the teacher. This indicates that when there is no teacher, the rapid infection of panic leads to a sharp increase in the movement speed of students. As the speed increases rapidly, students are jammed at the door at $14 \mathrm{~s}$, which leads to a decrease in evacuation speed after 14s. However, when there is a teacher, the evacuation speed remains stable and there is no congestion phenomenon when there is no teacher, so the evacuation time is shorter when there is a teacher than when there is no teacher.

It can be seen from figure 3 that the infection rate in the absence of a teacher is faster than that in the presence of a teacher, which is consistent with the changing trend of evacuation speed in figure 2. The rapid infection rate led to the rapid spread of panic, which made the students move faster, and the evacuation time was longer in the case of faster speed, which also confirmed the phenomenon of "fast is slow".

(2) Experiment 2: Different personality

In order to verify the influence of different personalities on emotional infection in evacuation process, experimental simulation was carried out on the basis of 50 agents. Figure 4 shows the emotional infection of open individuals $(\mathrm{O})$ with different proportions in the total number of people. The proportions of open individuals (O) are set as $20 \%$, $40 \%, 60 \%$ and $80 \%$ respectively.

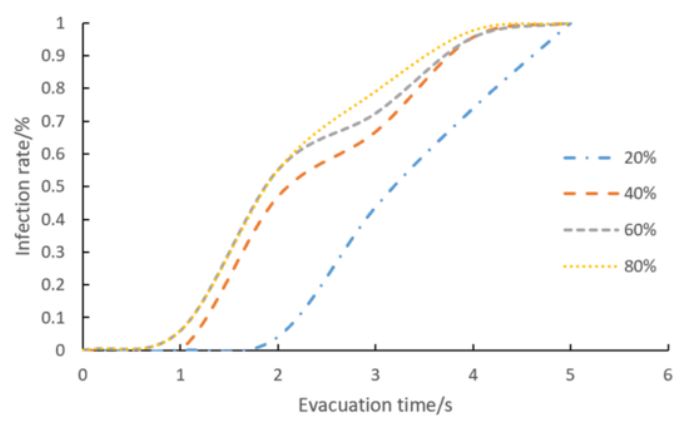

Figure 4. The influence of O-type individuals on emotional infection.

The results of figure 4 were analyzed: among the five types of individuals with different personalities, the open individuals had the largest receptivity to emotional infection and had a strong receptivity to emotional infection. With the increase of the proportion of open individuals, the infection time also accelerated. For example, when open individuals account for $20 \%$ and $80 \%$, when the proportion is $20 \%$, all individuals 
need $5 \mathrm{~s}$ to be infected; When the proportion is $80 \%$, it only takes $4 \mathrm{~s}$ for all individuals to become infected. It can be seen that different proportions of individual personalities have different effects on emotional infection, and play an important role in the process of emotional infection.

(3) Experiment 3: No administrator, different number of users

In this experiment, the number of evacuees was set as 10, 20, 30, 40 and 50, respectively, to study the influence of different numbers on emotional infection and evacuation efficiency.

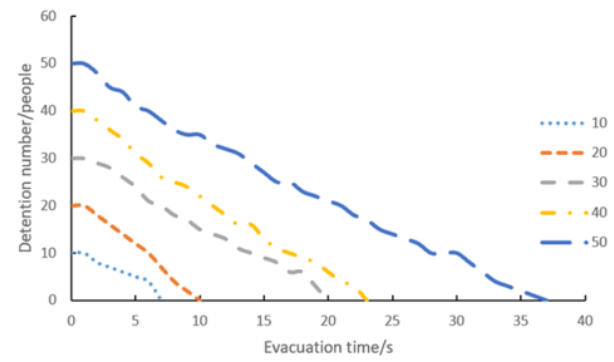

Figure 5. Influence of different number of people on evacuation efficiency

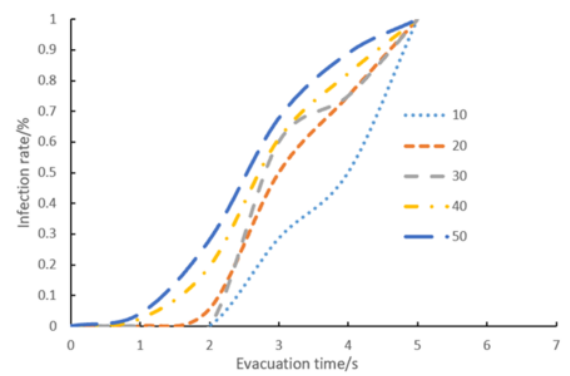

Figure 6. Influence of different number of people on emotional infection

Analysis of the results in figure 5: Different numbers of people have different influences on evacuation efficiency. With the increase of number of people, evacuation efficiency decreases and evacuation time increases. When the number of people is 10 , the evacuation time only needs $7 \mathrm{~s}$; When the number of people is 50 , the evacuation time is as long as $37 \mathrm{~s}$, so it can be seen that the more people, the lower the evacuation efficiency. Analysis of figure 6: It can be seen from figure 6 that, with the increase of the number of individuals, the speed of emotional infection actually slows down. This is because the fewer people there are, the larger the distance between individuals is, and the smaller the population density is, so the infection speed slows down, which is consistent with the reality.

\section{Conclusion}

Based on previous research models, this paper explores the simulation of classroom crowd behavior in emergency situations, and studies the crowd evacuation and emotional infection phenomena under the circumstances of management with and without 
administrators, different proportions of individual personalities and different numbers of individuals. The experiment shows that once an emergency occurs, the group emotion will rapidly develop into panic emotion, so regulating the group emotion, controlling the spread of panic emotion and stabilizing individual behavior can reduce the possibility of crowding accident. In the event of an emergency, there is a risk of crowding at the exit due to emotional infection. The presence of an administrator can improve the efficiency of evacuation and reduce risks, and also verify the phenomenon of "fast is slow". In addition, according to the results of evacuation time under different proportions of personality individuals, the more extroverted individuals, the faster the emotional infection rate, which verified the authenticity of the model. When the number of people in the classroom is different, the emotional infection speed and evacuation efficiency are also different. The more the number of people in the classroom, the faster the emotional infection speed and the lower the evacuation efficiency. Thus, controlling the number of people is beneficial to the stability of group mood. Future research should focus on the optimization of emotional infection models, such as the emotional sending ability of individuals with different personalities and the mechanism of emotional infection in other scenarios.

\section{References}

[1] Pengfei Zhao, Ahhong He. Research on interaction effect of Panic Emotion and Coping strategy of Trapped People in Major Coal Mine Accidents [J]. Coal Mine Safety, 201,52(09):261-264.

[2] Y Di. Study on emergency evacuation model of subway platform considering the impact of panic [D]. Beijing Jiaotong University,2017.

[3] Ruixiang Wang, Yinghua Zhang, Tingxin Qin, Jingjing Wang, Shiwei DING, Yukun Gao. Journal of Safety Science and Technology, 201,17(07):167-171.

[4] Mengxiao Cao, Guijuan Zhang, Lijun Huang, Hong Liu. Computer science,2017,44(06):306-311+316.

[5] Cuijuan Liu, Zhen Liu, Yanjie Chai, Tingting Liu, Zhongrui Ni. Journal of computer-aided design \& computer graphics,2020,32(04):660-670.

[6] Zhen Liu, Wei Jin, Peng Huang, Yanjie Chai. Journal of computer research and development,2013,50(12):2578-2589.

[7] Tingting Liu, Zhen Liu, Yanjie Chai, Cuijuan Liu, Jiaxin Chen. Journal of system simulation,2016,28(10):2448-2454.

[8] Lingli Zhang, Baoyun Wang, Ting Wang, Maolin Jiang. Statistics and analysis of high-risk sites of crowd crush accident [J]. Safety,2019,40(10):9-14.

[9] Liyun Dong, Dongkai Lan, Xiaoyin Duan. Modeling and simulation of crowd evacuation in dual-exit classroom [J]. Journal of Shanghai university (natural science),2018,24(02):217-224.

[10] Zhijian Chen, Yongxiang ZHAO. Simulation research on pedestrian evacuation in 3d lecture theatre based on social force model [J]. China safety science and technology,2020,16(04):5-11.

[11] Aisha Wang, Wei Peng. Study on evacuation of classroom in emergency [J]. China safety science and technology,2012,8(08):163-166.

[12] Lijun Ran, Mao Liu. Journal of Safety and Environment,2007(04):135-138. 